\title{
Monte Carlo Studies of the Brownian Motion of a Polymer Chain under Topological Constraints
}

\author{
Masao DoI \\ Department of Applied Physics, Faculty of Engineering, \\ The University of Tokyo, Bunkyo-ku, Tokyo, Japan.
}

(Received May 12, 1973)

\begin{abstract}
Monte Carlo Studies are made to examine the validity of the de-Gennes' theory of the stochastic motion of a polymer chain in the presence of fixed obstacles. The two-dimensional cases are treated. The topological requirement that the chain cannot intersect any of the obstacles is imposed on the stochastic motion. Observations are made on the diffusion coefficient of the center of mass, the relaxation time of the end-to-end vector and the mean-square displacement of a monomer, by varying the chain length and the concentration of the obstacles. The results are compared with those of de-Gennes' theory and Rouse's. It is found that de-Gennes' theory provides a reasonable explanation for the slow relaxation phenomena under topological restrictions. Some minor revisions are made to obtain better agreement. It is found that, for the fast relaxation phenomena, the agreement is not good even if the concentration of the obstacles is sufficiently large. The condition for the applicability' of the de-Gennes' theory is also discussed. The transition from the Rouse-type motion to the de-Gennes-type motion is observed and found to be rather diffuse.

KEY WORDS Monte Carlo Study / Topological Constraint / Entanglement Effect / de-Gennes' Theory / Rouse's Theory /
\end{abstract}

Although the importance of the entanglement effect has been well appreciated in concentrated polymer solutions and in polymer melts, the theoretical understanding of the effect has remained rather inadequate untill recently. ${ }^{1}$ The theoretical difficulty lies in the problem of how to describe the entanglement effect. Bueche, ${ }^{2}$ Grasseley, ${ }^{3}$ and Chikahisa ${ }^{4}$ have treated the undiluted polymer solution case based upon the hypothesis that spheres of the macromolecules moving in the solvent exert some frictional forces on each other. Chompff, Duiser ${ }^{5}$ and Prins, ${ }^{6}$ Hayashi, ${ }^{7}$ and Yamamoto ${ }^{8}$ considered the polymer chain in the undiluted solution as a Rouse chain having low-mobility points. Further Yamamoto $^{9}$ proposed a constitutive equation of concentrated polymer solutions based upon the temporary cross-linkage model. Although some of the authors have succeeded in explaining the experimental results, these models cannot be expected to give a fundamental understanding of the entanglement effect, since they do not take into account the topological nature of the entanglement effect. An exact mathemat- ical method to describe the permanent topological constraints was proposed by Edwards. ${ }^{10}$ However the results are so complicated that such an approach hardly seems applicable to the dynamical problems.

Recently, however, an illuminating theory was proposed by de-Gennes ${ }^{11}$ who showed a convenient way of handling the topological restrictions. The problem he considered is a rather simple one compared to that of the real concentrated polymer solutions. He considered a stochastic motion of a polymer chain in fixed three-dimensional networks. Although such a system is far from the real concentrated polymer chain system, the topological requirement that the chains cannot interest each other is taken into account exactly. The essential point in his theory is that the motion of the polymer chain in networks can be understood as a "reptation process" in some virtual tube. The virtual tube represents the topological restriction imposed on the chain. In short time scale, the polymer is confined in some virtual tube, but in sufficiently long time scale, the polymer diffuses from one 
virtual tube to another by the reptation process. Let us call this model as "reptation model" in comparison to the "bead-spring model" which describe the stochastic motion in free space.

By taking the above view point, de-Gennes showed that many important dynamic properties are different in the presence of the fixed obstacles compared to those in free space. The main differences are as follows.

(1) The diffusion coefficient of the center of mass is proportional to $N^{-1}$ in the bead-spring model, but is proportional to $N^{-2}$ in the reptation model, where $N$ is the degree of polymerization.

(2) The longest relaxation time of the correlation function of the end-to-end vector is proportional to $N^{2}$ in the bead-spring model, whereas it is proportional to $N^{3}$ in the reptation model.

(3) The mean-square displacement of a monomer in time $t$ is proportional to $t^{1 / 2}$ and $t^{1 / 4}$ in the bead-spring model and in the reptation model, respectively.

The characteristic differences in these models are so clear that it is interesting to confirm these results. Since we consider that de-Gennes' theory proposes an important idea for the investigation of concentrated polymer solutions, we suppose it is worthwhile studying the validity of his theory. In this paper we carried out a Monte Carlo study of the stochastic motion of a chain in the presence of fixed obstacles.

Our interest lies in the following points. Since the concept of the virtual tube has meaning only when the interval of the network is short enough compared to the length of the polymer chain, the reptation model should be applicable only for sufficiently dense networks or for sufficiently long polymer chains. On the other hand for sufficiently low concentrations or sufficiently short chains, the bead-spring model will be a good approximation. A question then arises; in what circumstance the reptation model or beadspring model is applied and further what will be the dynamical behaviour of the chain in the case of the intermediate concentration and chain length. The latter point is of interest since it is well known that, in real concentrated polymer solutions, the molecular-weight dependency of the viscosity shows an abrupt change as the molecular-weight increases. To investigate these points, the stochastic motion of the polymer chain was studied by changing the chain length and the concentration of the obstacles.

Our computer simulation is a very simple one. We consider a motion of a polymer chain in the two-dimensional plane. Instead of the threedimensional networks, we set up piles perpendicularly to the plane as obstacles and impose the topological requirement on the stochastic motion that the chain cannot intersect the piles. The algolism of the stochastic motion is so chosen as the motion is coincident with that of the bead-spring model in the absence of the piles. Observations are made on the following quantities; the position of the center of mass;

$$
\boldsymbol{R}_{\mathrm{G}}=\frac{1}{N} \sum_{m=1}^{N} \boldsymbol{R}_{n}
$$

where $\boldsymbol{R}_{n}$ is the position vector of the $n$-th monomer and $N$ is the number of the monomers, the end-to-end vector;

$$
\boldsymbol{P}=\boldsymbol{R}_{N}-\boldsymbol{R}_{1}
$$

and the position of the (N/2)-th monomer, which we shall call the center monomer;

$$
\boldsymbol{R}_{\mathrm{c}}=\boldsymbol{R}_{(N / 2)}
$$

Time correlation functions of these quantities are calculated as the mean-square displacement of the center of mass and the center monomer;

$$
\left\langle\left(\boldsymbol{R}_{\mathrm{G}}(t)-\boldsymbol{R}_{\mathrm{G}}(0)\right)^{2}\right\rangle, \quad\left\langle\left(\boldsymbol{R}_{\mathrm{c}}(t)-\boldsymbol{R}_{\mathrm{c}}(0)\right)^{2}\right\rangle
$$

and the time correlation function of the end-toend vector;

$$
\langle\boldsymbol{P}(t) \cdot \boldsymbol{P}(0))\rangle
$$

These correlation functions are obtained by varying the density of the piles and the length of the chain, and compared with the theories. In the following section the theoretical results are summarized.

\section{THEORIES}

First, consider the bead-spring model. As is well known the mean-square displacement of the center of mass is given by

$$
\begin{gathered}
\left\langle\left(\boldsymbol{R}_{\mathrm{G}}(t)-\boldsymbol{R}_{\mathrm{G}}(0)\right)^{2}\right\rangle=4 D_{\mathrm{G}} t \\
D_{\mathrm{G}}=\frac{D}{N}
\end{gathered}
$$


where $D$ is the diffusion coefficient of a monomer $\left(D=k_{\mathrm{B}} T / \zeta ; \zeta\right.$ is the friction coefficient of a monomer). In eq 4 , the factor 4 is necessary since we are considering two-dimensional motion. The time correlation function of the end-to-end vector is given by

$$
\begin{gathered}
\langle\boldsymbol{P}(t) \cdot \boldsymbol{P}(0)\rangle=\sum_{k \text { odd }} \frac{8}{N \alpha_{k}} \mathrm{e}^{-2 D \alpha_{k} t} \\
\alpha_{k}=\frac{4}{a^{2}} \sin ^{2} \frac{k \pi}{2 N}
\end{gathered}
$$

where $a$ is the bond length. For sufficiently large $t$, eq 6 is approximated as

$$
\begin{gathered}
\langle\boldsymbol{P}(t) \cdot \boldsymbol{P}(0)\rangle=\frac{8 N a^{2}}{\pi^{2}} \mathrm{e}^{-t / \tau_{\mathrm{R}}} \\
\tau_{\mathrm{R}}=\frac{a^{2} N^{2}}{2 D \pi^{2}}
\end{gathered}
$$

The mean-square displacement of a monomer, for which we have chosen the center monomer, was calculated by de-Gennes ${ }^{12}$ for the threedimensional case. The similar calculation for the two-dimensional case leads to the result

$$
\left\langle\left(\boldsymbol{R}_{\mathrm{c}}(t)-\boldsymbol{R}_{\mathrm{c}}(0)\right)^{2}\right\rangle= \begin{cases}2 a \sqrt{\frac{2 D t}{-\pi}} & \left(t \lesssim \tau_{\mathrm{R}}\right) \\ 4 \frac{D}{N} t & \left(t \geq \tau_{\mathrm{R}}\right)\end{cases}
$$

Next, let us summarize the results of deGennes' theory. The diffusion of the center of mass is predicted as

$$
\begin{gathered}
\left\langle\left(\boldsymbol{R}_{\mathrm{G}}(t)-\boldsymbol{R}_{\mathrm{G}}(0)\right)^{2}\right\rangle=4 D_{\mathrm{G}} t \\
D_{\mathrm{G}}=\frac{\Delta \bar{\rho} b^{2}}{2 a N^{2}}
\end{gathered}
$$

where $\Delta$ is the diffusion coefficient of the "defect" and $\bar{\rho}$ is the "equilibrium density of defect" and $b$ is the "stored length of the defect" in de-Gennes' terminology. At this point, it should be mentioned that there is an error in eq III 7 of de-Gennes' paper. Since $\left\langle P_{z}^{2}\right\rangle=N a^{2} / 3$ in the equation, it should be corrected as

$$
\mu_{\mathrm{tot}}=\frac{\mu \bar{\rho} b^{2}}{3 N a^{2}}
$$

In our case the factor $1 / 2$ should be chosen instead of $1 / 3$ because of the two-dimensional motion, and thus we obtain eq 12 .
The correlation function of the end-to-end vector is given as follows for sufficiently long time.

$$
\langle\boldsymbol{P}(t) \cdot \boldsymbol{P}(0)\rangle=\frac{8 N a^{2}}{\pi^{2}} \mathrm{e}^{-t / \tau_{\mathrm{D}}}
$$

where

$$
\tau_{\mathrm{D}}=\frac{(N a)^{3}}{\pi^{2} \bar{\rho} b^{2} \Delta}
$$

Finally the mean-square displacement of a monomer is given as

$$
\left\langle\left(\boldsymbol{R}_{\mathrm{c}}(t)-\boldsymbol{R}_{\mathrm{c}}(0)\right)^{2}\right\rangle= \begin{cases}\frac{2}{\pi^{3 / 4}} a b \bar{\rho}(\Delta \cdot t)^{1 / 4} & \left(t \lesssim T_{\mathrm{d}}\right) \\ 4 D_{\mathrm{G}} t & \left(t \geq T_{\mathrm{d}}\right)\end{cases}
$$

with

$$
T_{\mathrm{d}}=\frac{(N a)^{2}}{\Delta \pi^{2}}
$$

where $D_{G}$ is given by eq 12 .

Although eq 14 and 16 are proposed for the. three-dimensional motion, they can be applied for the two-dimensional case without any modification. This is easily verified by examining the logical construction of de-Gennes' theory.

\section{MODEL}

The model taken in our computer simulation is illustrated in Figure 1. The polymer chain is replaced on the two-dimensional square lattice. Piles are set up regularly in the center of the lattice points. The stochastic motion of the chain is caused by the random transition of the position of the monomers. The transition in which the chain intersects the piles is inhibited.

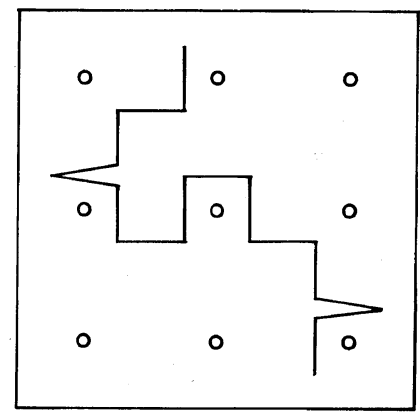

Figure 1. Model taken in the computer simulation. The circles denote the piles set up vertically to the plane. 
The procedure causing transition of a monomer is as follows.

(1) A monomer is selected randomly. Let us denote its position by $\boldsymbol{R}_{n}$.

(2) This monomer is replaced to a new position $\boldsymbol{R}_{n}{ }^{\prime}$ by the following rule.

a) If the monomer is not at the chain ends, that is, if $n \neq 1$ or $N$, it is put to

$$
\boldsymbol{R}_{n}{ }^{\prime}=\boldsymbol{R}_{n+1}+\boldsymbol{R}_{n-1}-\boldsymbol{R}_{n}
$$

unless the chain does not intersects the piles. If it does, the transition is not made.

b) If the monomer is at the chain end, that is, if $n=1$ or $N$, a new direction of the bond vector $\boldsymbol{a}$ is selected randomly and the monomer is put to

$$
\boldsymbol{R}_{1}^{\prime}=\boldsymbol{R}_{2}-\boldsymbol{a}
$$

or

$$
\boldsymbol{R}_{N}{ }^{\prime}=\boldsymbol{R}_{N-1}+\boldsymbol{a}
$$

irrespectively of the piles.

We call this process "bead cycles" and further we call $N$ bead cycles "cycle." As will be shown below it is the cycle which plays a role of the time scale in the real world. In our computer simulation, time $t$ means $t$ cycles, in which $t N$ selections of monomers are made.

The algolism given above is the same as used by Verdier and Stockmayer ${ }^{13}$ in case where there are no piles. It is exactly proved ${ }^{14}$ that, in that case, the stochastic motion shows the same behaviour predicted by the Rouse theory or the bead-spring model. We can easily determine the parameters appearing in that theory. In our calculation the lattice constant is chosen as the unit of length. Therefore the bond length is equal to unity.

$$
a=1
$$

To determine the diffusion constant of the monomer, let us calculate the translational diffusion constant of the center of mass. From eq 4

$$
D_{\mathrm{G}}=\frac{1}{4 t}\left\langle\left(\boldsymbol{R}_{\mathrm{G}}(t)-\boldsymbol{R}_{\mathrm{G}}(0)\right)^{2}\right\rangle
$$

where $\langle\cdots\rangle$ denotes the average with respect to the random process of the transition and the initial configuration of the polymer chain. By

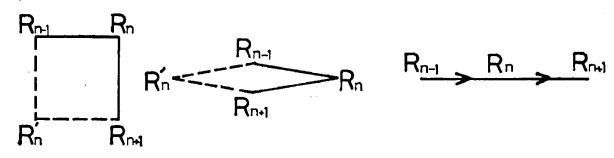

Figure 2. Three types of the elementaly transition.

setting $t=1 / N$ (=one bead cycle), we obtain

$$
\begin{aligned}
D_{\mathrm{G}} & =\frac{N}{4}\left\langle\left(\boldsymbol{R}_{\mathrm{G}}{ }^{\prime}-\boldsymbol{R}_{\mathrm{G}}\right)^{2}\right\rangle=\frac{N}{4}\left\langle\frac{1}{N^{2}}\left(\boldsymbol{R}_{n}{ }^{\prime}-\boldsymbol{R}_{n}\right)^{2}\right\rangle \\
& =\frac{1}{4 N}\left\langle\left(\boldsymbol{R}_{n}{ }^{\prime}-\boldsymbol{R}_{n}\right)^{2}\right\rangle
\end{aligned}
$$

where $\boldsymbol{R}_{n}$ and $\boldsymbol{R}_{n}{ }^{\prime}$ are the initial and final positions of the monomer which makes the transition during this time. The length $\left|\boldsymbol{R}_{n}-\boldsymbol{R}_{n}{ }^{\prime}\right|$ can take the values $\sqrt{2}, 2$ or 0 , corresponding to the three types of the local conformation of the chain shown in Figure 2. The statistical weight of these conformations are $1 / 2,1 / 4$ and $1 / 4$ respectively. Therefore we obtain

$$
\begin{aligned}
D_{G} & =\frac{1}{4 N}\left(\frac{1}{2} \times(\sqrt{2})^{2}+\frac{1}{4} \times 2^{2}\right) \\
& =\frac{1}{2 N}
\end{aligned}
$$

Then the diffusion coefficient of a monomer is given

$$
D=\frac{1}{2}
$$

Note that if we take the "bead cycle" as the unit of the time scale, the diffusion coefficient of a monomer depends on the molecular-weight $N$. This is the reason why we take "cycle" as the unit of the time scale.

Let us rewrite the theoretical results of the bead-spring model by using the above parametrization. By putting $a=1$, and $D=1 / 2$, we obtain

$$
\begin{gathered}
\left\langle\left(\boldsymbol{R}_{\mathrm{G}}(t)-\boldsymbol{R}_{\mathrm{G}}(0)\right)^{2}\right\rangle=4 D_{\mathrm{G}} t \\
D_{\mathrm{G}}=\frac{1}{2 N} \\
\langle\boldsymbol{P}(t) \cdot \boldsymbol{P}(0)\rangle=\frac{8 N}{\pi^{2}} \mathrm{e}^{-t / \tau_{\mathrm{R}}} \\
\tau_{\mathrm{R}}=\frac{N^{2}}{\pi^{2}} \\
\left\langle\left(\boldsymbol{R}_{\mathrm{c}}(t)-\boldsymbol{R}_{\mathrm{c}}(0)\right)^{2}\right\rangle= \begin{cases}\frac{2}{\sqrt{\pi}} t^{1 / 2} & \left(t \lesssim \tau_{\mathrm{R}}\right) \\
\frac{2}{N} t & \left(t \gtrsim \tau_{\mathrm{R}}\right)\end{cases}
\end{gathered}
$$


Such complete parametrization cannot be made for de-Gennes' theory since there appear undetermined parameters such as $\bar{\rho}, b$ and $\dot{\Delta}$. Therefore we focus our attention on the following characteristic points of the results

$$
\begin{gathered}
\left\langle\left(\boldsymbol{R}_{\mathrm{G}}(t)-\boldsymbol{R}_{\mathrm{G}}(0)\right)^{2}\right\rangle=4 D_{\mathrm{G}} t \\
D_{\mathrm{G}} \propto N^{-2} \\
\langle\boldsymbol{P}(0) \cdot \boldsymbol{P}(t)\rangle=\frac{8 N}{\pi^{2}} \mathrm{e}^{-t / \tau}{ }_{\mathrm{R}} \\
\tau_{\mathrm{R}} \propto N^{3} \\
\left\langle\left(\boldsymbol{R}_{\mathrm{c}}(t)-\boldsymbol{R}_{\mathrm{c}}(0)\right)^{2}\right\rangle \propto \begin{cases}t^{1 / 4} & \left(t \lesssim T_{\mathrm{d}}\right) \\
t & \left(t \gtrsim T_{\mathrm{d}}\right)\end{cases}
\end{gathered}
$$

However there exists an important relation which can be checked in the computer simulation. Substituting eq 12 into eq 15 , we obtain

$$
D_{\mathrm{G}} \cdot \tau_{\mathrm{D}}=\frac{N a}{2 \pi^{2}}=\frac{N}{2 \pi^{2}}
$$

These theoretical results of eq 26-36 can be checked by the computer experiments.

\section{METHOD OF THE COMPUTER SIMULATION}

In our computer experiments, the interval between the piles are chosen as 2,3 and infinity, therefore the concentration of the piles respectively becomes, $c=1 / 2^{2}=0.25, c=1 / 3^{2}=0.11$ and $c=0.0$. The length of the polymer chain is chosen, $N=8,12,16,20,24,32$ and 40 .

Calculation of the correlation function is carried out by the following procedure. First the initial configuration of the polymer chain is given by sequentially selecting the direction of the bond vectors randomly. The stochastic motion is then caused in the way explained in the foregoing section. At intervals of $1-10$ cycles the vectors $\boldsymbol{R}_{\mathrm{G}}, \boldsymbol{P}$ and $\boldsymbol{R}_{\mathrm{c}}$ are calculated and recorded. The stochastic motion is continued for $400 \tau_{\mathrm{R}}-200 \tau_{\tau_{\mathrm{R}}}$ cycles, where $\tau_{\mathrm{R}}$ is given by eq 29 . After that, the time correlation function is calculated as follows

$$
\begin{array}{r}
\left\langle\left(\boldsymbol{R}_{\mathrm{G}}(t)-\boldsymbol{R}_{\mathrm{G}}(0)\right)^{2}\right\rangle \\
=\frac{1}{N_{\mathrm{s}}-t} \sum_{j=1}^{N_{\mathrm{s}}-t}\left(\boldsymbol{R}_{\mathrm{G}}(t+j)-\boldsymbol{R}_{\mathrm{G}}(j)\right)^{2} \\
\langle\boldsymbol{P}(t) \cdot \boldsymbol{P}(0)\rangle=\frac{1}{N_{\mathrm{s}}-t} \sum_{j=1}^{N_{\mathrm{S}}-t} \boldsymbol{P}(t+j) \cdot \boldsymbol{P}(j)
\end{array}
$$

$$
\begin{aligned}
& \left\langle\left(\boldsymbol{R}_{\mathrm{c}}(t)-\boldsymbol{R}_{\mathrm{c}}(0)\right)^{2}\right\rangle \\
& =\frac{1}{N_{\mathrm{s}}-t} \sum_{j=1}^{N_{\mathrm{s}}-t}\left(\boldsymbol{R}_{\mathrm{c}}(t+j)-\boldsymbol{R}_{\mathrm{c}}(j)\right)^{2}
\end{aligned}
$$

where $N_{\mathrm{s}}$ is the number of the samples recorded. We call this procedure as one "experiment." The experiments are repeated three (or two for $N=32$ and 40) times for each concentration of piles and molecular weight of the chain by alternating the initial configuration of the chain and the initial value of the pseudo random number.

Our choice of the computation time of one experiment is based upon the following consideration. We may assume $\tau_{R}$ is the time in which the effect of the initial conformation of the chain vanishes. Therefore the samples obtained from the sequential stochastic motion in $T$ cycles are equivalent to those obtained by the $T / \tau_{\mathrm{R}}$-independent simulations. The statistical error of the latter is of the order $\left(T / \tau_{\mathrm{R}}\right)^{-1 / 2}$. To keep this error within $5 \%$, we should put $T=400 \tau_{\mathrm{R}}$. The above consideration is based upon the case $c=$ 0.0. For larger concentration, the computation time must be put longer than this, since the relaxation time becomes longer. The computation time was chosen as $T=1000 \tau_{\mathrm{R}}$ (the case of $c=$ 0.11 ) and $T=2000 \tau_{\mathrm{R}}$ (the case of $c=0.25$ ).

The computer program is written in FORTRAN and FASP. The computation is carried out by using FACOM 270/20 and FACOM 270/30. The program is made out so as to show the motion of the chain in the graphic display whenever it is demanded. In Figure 3, several aspects of the stochastic motion of the chain are shown.

\section{RESULTS}

The Mean-Square Displacement of Center of Mass

In Figure 4, a typical result of the mean-square displacement of center of mass is shown. It is observed that, for the case of $c=0.25,\left\langle\left(\boldsymbol{R}_{\mathrm{G}}(t)-\right.\right.$ $\left.\left.\boldsymbol{R}_{G}(0)\right)^{2}\right\rangle$ is not proportional to $t$. Instead the curve is well fitted by the following equation

$$
\left\langle\left(\boldsymbol{R}_{\mathrm{G}}(t)-\boldsymbol{R}_{\mathrm{G}}(0)\right)^{2}\right\rangle=A+B t
$$

For the case $c=0.0, A$ is equal to zero, but in general $A$ does not vanish, which can also be clearly observed in other data. This fact was not predicted in de-Gennes' paper. However 

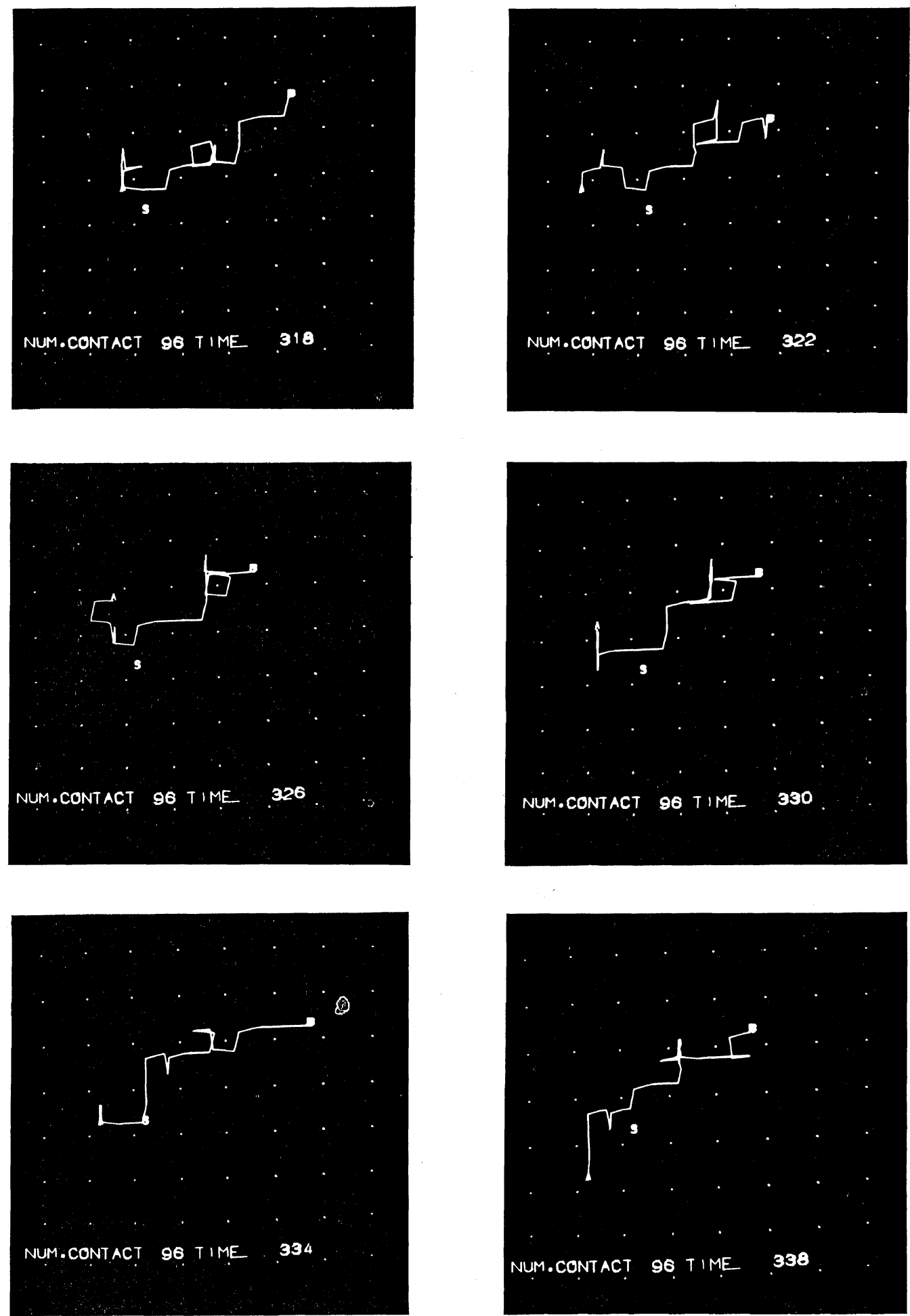

Figure 3. Typical behaviour of the stochastic motion of the chain. 
M. DoI
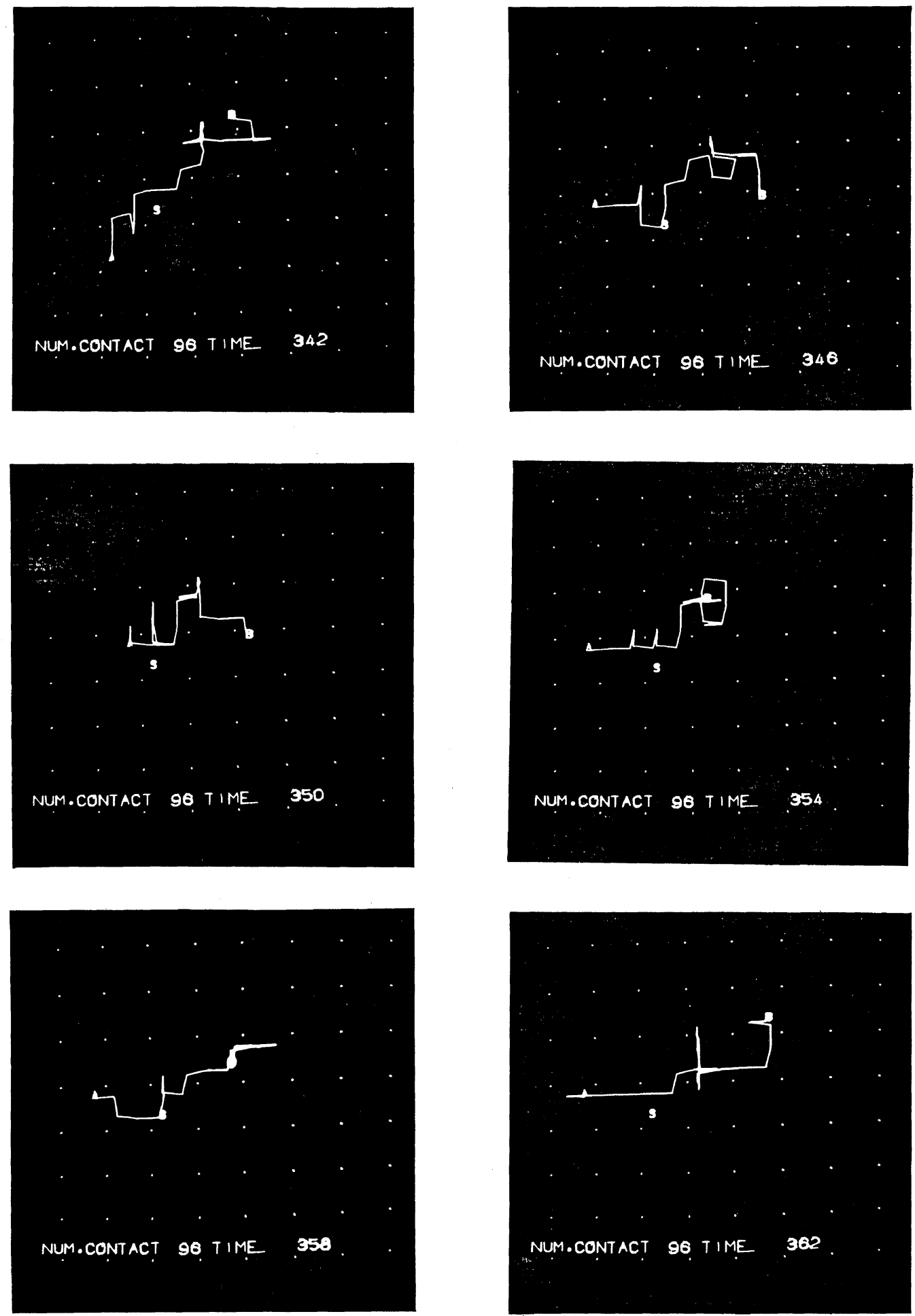

Figure 3 (continued). Typical behaviour of the stochastic motion of the chain. 


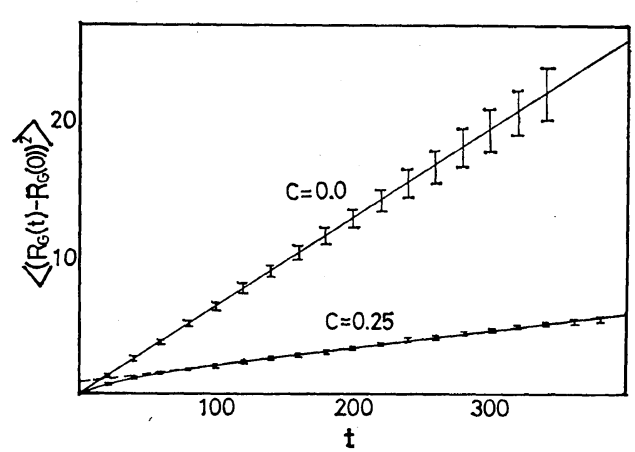

Figure 4. Mean-square displacement of the center of mass is plotted against time, for the case of $c=0.0, N=32$ and $c=0.25, N=32$.

it is not a defect of the reptation model, but, it may be a consequence of the mathematical approximation adopted in his paper. In fact a more careful treatment of the reptation model gives rise the nonvanishing value of $A$, as discussed in Appendix I.

The diffusion coefficient of center of mass is given by the slope of the graph:

$$
D_{G}=\frac{1}{4} B
$$

In Figure 5 the molecular-weight dependency of the diffusion coefficient is shown, where $N^{2} D_{\mathrm{G}}$ is plotted against $N$ for convenience of comparison with eq 27 and 32 .

It is clearly seen that the curve for $c=0.0$ is a straight line the slope of which is 0.5 . This exactly agrees with eq 27 . However it is a necessary consequence because we have defined $D_{\mathrm{G}}$ by eq 24 or 27 . Then it should be said that the

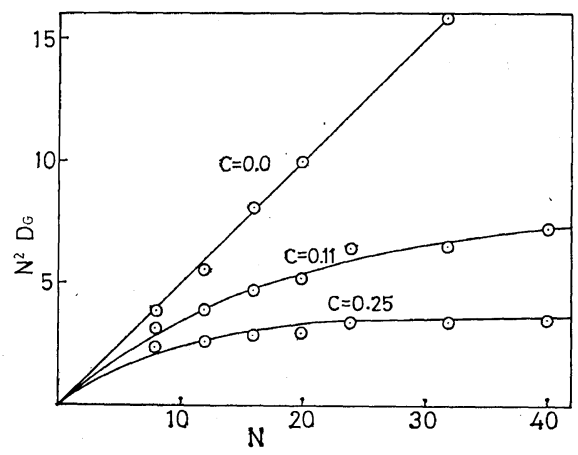

Figure 5. Diffusion coefficient multiplied by $N^{2}$ is plotted against the molecular weight $N$. agreement confirms the validity of our computer simulation.

On the other hand, it is observed that the curve for $c=0.25$ becomes a horizontal straight line for $N \geqq 20$. This provides clear evidence of the validity of the de-Gennes' result of eq 32 . Further the curve $c=0.11$ lies between those two curves and the slope decreases as ' $N$ increases. For sufficiently large values of $N$, the slope seems to reach zero. This means that the reptation model becomes applicable for a sufficiently long chain even if the density of the network is very small. This is an expected result. Further it is concluded that the limiting value of $N^{2} D_{G}$ for large $N$ depends on the concentration of the piles. This means that the parameter appearing in the reptation model such as $\bar{\rho}, b$ and $\Delta$ are the function of the concentration of the network. This is a reasonable result though at this stage we cannot predict the concentration dependency of the parameters.

The Correlation Function of the End-To-End Vector

In Figure 6, a result of the time correlation function of the end-to-end vector is shown in semilogarithmic scale. It is observed at first that the mean-square end-to-end distance is independent of the concentration of the piles and equals to

$$
\left\langle\boldsymbol{P}^{2}\right\rangle=N-1
$$

This fact can be observed generally in other data. This is a consequence of the fact that the existence of the piles does not change the equilibrium distribution function of the conformation of the polymer chain. This is a charac-

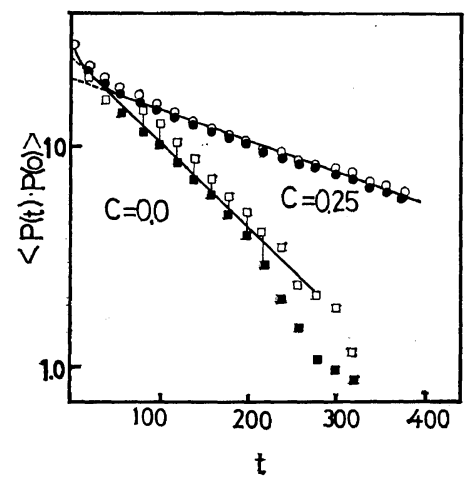

Figure 6. Time correlation functions of the endto-end vector is plotted against time for the case of $c=0.0, N=32$ and $c=0.25, N=32$. 
teristic feature of the entanglement effect; the entanglement effect is important only for the dynamic properties, and does not affect the static properties.

The time correlation function is well fitted by the following equation

$$
\langle\boldsymbol{P}(t) \cdot \boldsymbol{P}(0)\rangle=\varepsilon \mathrm{e}^{-t / \tau}
$$

except for the short time scale (more precisely excepting the time $t \leqslant \tau / 9$ as is seen from eq 6). On the other hand it is observed that the experimental result deviates in the long time scale in the graph of $c=0.0$. This arises from the fact that, for large values of $t$, the true value of $\langle\boldsymbol{P}(t) \cdot \boldsymbol{P}(0)\rangle$ becomes so small that the statistical deviation becomes of importance. Therefore we must fit the curve with eq 43 in some appropriate time region. By this fitting process we obtain the longest relaxation time $\tau$ and the corresponding relaxation strength $\varepsilon$.

Let us first consider the relaxation strength. Both the Rouse theory and de-Gennes' predict that $\varepsilon$ has constant value equal to $8 N / \pi^{2}$. However as can be seen in Figure $6, \varepsilon$ is smaller than this value for $c=0.25$. In Figure $7, \varepsilon$ is plotted against $N$. It is seen that $\varepsilon$ becomes smaller as the molecular weight increases and tends to some definite value for $c=0.25$ and 0.11 . For the case $c=0.25$, this limiting value is 0.8 times of the theoretical value. However this discrepancy is not a crucial one for the reptation model. In fact it is shown that the discrepancy originates from the approximation adopted in the de-Gennes' calculation. In his calculation it is assumed that the tube length $L=N a(1-\rho b)$, which is the length of the part of the virtual tube enveloping the chain, does not

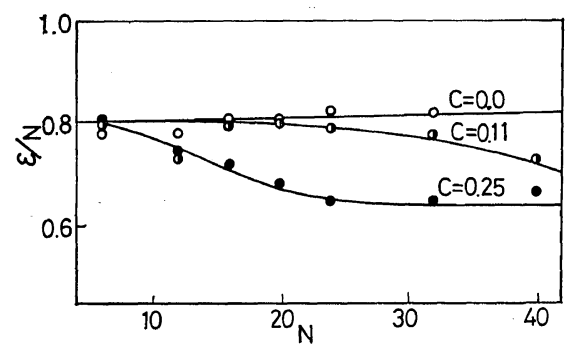

Figure 7. Relaxation strength of the longest relaxation time is plotted against the molecular weight $N$. fluctuate and is equal to

$$
L=N a
$$

We consider that this approximation is not good in our case. In fact, if the fluctuation of the tube length is taken into account, consistent results can be recovered. If we consider the fluctuation of the tube length, eq 8 and 9 are corrected as

$$
\begin{aligned}
\varepsilon & =\frac{8 N a^{2}}{\pi^{2}}(1-\bar{\rho} b) \\
\tau_{\mathrm{D}} & =\frac{(N a)^{3}}{\pi^{2} \bar{\rho} b^{2} \Delta}(1-\bar{\rho} b)^{2}
\end{aligned}
$$

Equation 46 is immediately obtained by substituting $N a(1-\bar{\rho} b)$ instead of $N a$ in eq V 12 of de-Gennes' paper. Equation 45 is obtained from the following consideration. The decay of the time correlation of the end-to-end vector occurs mainly by a diffusion process along the tube, but it also arises from fluctuation of the tube length. The relaxation time of the latter process is of the order of $T_{\mathrm{d}}$, which is much shorter compared to $\tau_{\mathrm{D}}$, and its relaxation strength is $\bar{\rho} b$ times of the total relaxation strength. Therefore, for the vector correlation function $G_{n m}(t)=$ $\left\langle\boldsymbol{a}_{n}(t) \cdot \boldsymbol{a}_{m}(0)\right\rangle$, which describes only the diffusion process in de-Gennes' paper, we must impose the following initial condition

$$
G_{n m}(0)=(1-\bar{\rho} b) \delta_{n m}
$$

This correction leads to eq 45 . Comaring eq 45 with the observed value of $\varepsilon$, we can say $\bar{\rho} b$ is equal to 0.2 for the case $N \geqslant 20, c=0.25$. Later we shall show that this value is a reasonable one.

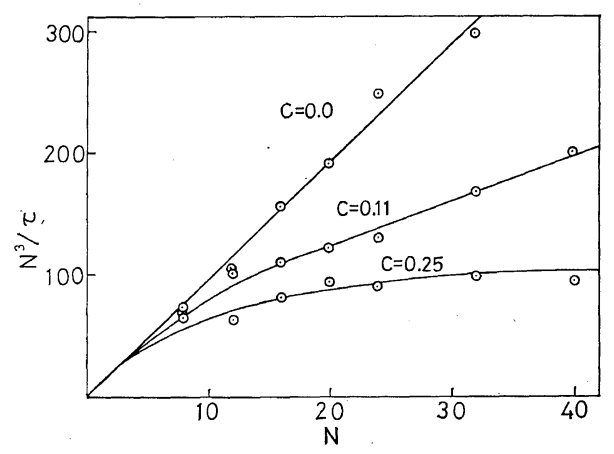

Figure 8. Molecular-weight dependency of the relaxation time $\tau$ of the end-to-end vector, where $N^{3} / \tau$ is plotted against $N$.

Polymer J., Vol. 5, No. 3, 1973 
Let us now turn to the relaxation time. In Figure $8, N^{3} / \tau$ is plotted against $N$ for convenience of comparison with eq 29 and 34 . For the case $c=0.0$, the curve is a straight line the slope of which is 9.8 , which is in good agreement with the theoretical value $\pi^{2} \cong 9.86$. This also provides a cross-check of the validity of our computer simulation as discussed in connection with $D_{G}$. The curve for $c=0.25$ is a horizontal straight line for $N \gtrsim 20$. This result also provides further clear evidence of de-Gennes' theory. Note that the value of $N$, at which the slope of the curve becomes zero, is consistent for both cases of $N^{2} D_{G}$, and $N^{3} / \tau$. The curve for $c=0.11$ in Figure 8 is also similar to that shown in Figure 5, except that the slope seems slightly larger in Figure 8 than in Figure 5. In conclusion, the data for the relaxation times confirm the conclusion drawn from the data for the diffusion coefficient.

Let us now verify eq 30 . In Table I, the value $D_{\mathrm{G}} \tau / N$ is tabulated for various values of $N$. Both the Rouse theory and de-Gennes' predict that this value is equal to $1 / 2 \pi^{2} \cong 5.06 \times 10^{-2}$. The agreement is good for $c=0.0$ but not so good for $c=0.11$ and $c=0.25$. However if we use eq 40 , the theoretical value is corrected as.

$$
\frac{D_{\mathrm{G}} \tau}{N}=\frac{(1-\bar{\rho} b)^{2}}{2 \pi^{2}}
$$

For the case $c=0.25$ and $N \geqslant 20, \bar{\rho} b$ has been obtained from the data of the relaxation strength as $\bar{\rho} b=0.2$. Using this value, we obtain

$$
\frac{D_{\mathrm{G}} \tau}{N} \cong 3.3 \times 10^{-2} \quad(c=0.25, N \gtrsim 20)
$$

then the agreement between the theory and experiment becomes very good.
Mean-Square Displacement of the Center Monomer

In Figure 9 the mean square displacement of the center monomer in time $t$ is shown against $t$, where $\ln \left\langle\left(\boldsymbol{R}_{\mathrm{c}}(t)-\boldsymbol{R}_{\mathrm{c}}(0)\right)^{2}\right\rangle$ is plotted against $\ln t$. The curve for $c=0.0$ agrees completely with the theoretical prediction of eq 30 . The curve for $c=0.25$ is qualitatively in agreement with eq 35 . However the slope of the curve for the short time is not equal to one-fourth but larger than this. To investigate this point, we define $\alpha$ by the slope of the curve for $t \lesssim T_{\mathrm{d}}$, that is, we assume

$$
\left\langle\left(\boldsymbol{R}_{\mathrm{c}}(t)-\boldsymbol{R}_{\mathrm{c}}(0)\right)^{2}\right\rangle \propto t^{\alpha}
$$

At this stage we cannot give any theoretical basis for our assumption of eq 50 . However the quantity $\alpha$ is determined fairly accurately from the experimental data. In Figure 10, the molecular weight dependency of $\alpha$ is shown. It is immediately seen that the value $\alpha$ is much larger than the theoretical value even for large molecular-weights and large concentrations. As the molecular-weight increases, the value $\alpha$ tends

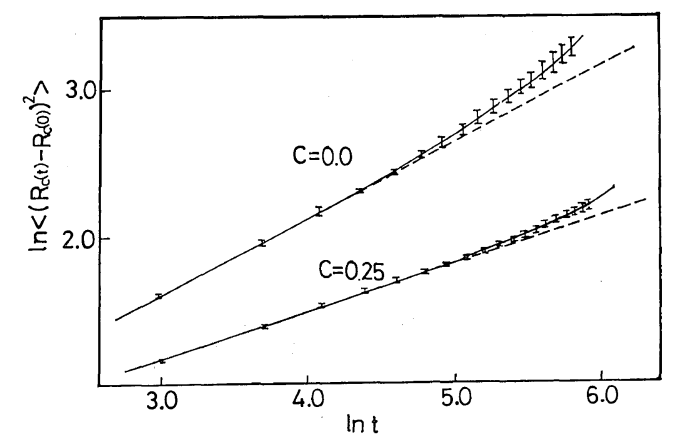

Figure 9. Mean square displacement of the center monomer is plotted against time for the case of

\begin{tabular}{|c|c|c|c|c|c|c|c|c|c|}
\hline \multirow{2}{*}{$N$} & \multicolumn{3}{|c|}{$c=0.0$} & \multicolumn{3}{|c|}{$c=0.11$} & \multicolumn{3}{|c|}{$c=0.25$} \\
\hline & $D_{\mathrm{G}}, \times 10^{2}$ & $1 / \tau, \times 10$ & $\tau D_{\mathrm{G}} / N, \times 10$ & $D_{G}, \times 10^{2}$ & $1 / \tau, \times 10$ & $\tau D_{G} / N, \times 10^{2}$ & $D_{\mathrm{G}}, \times 10^{2}$ & $1 / \tau, \times 10$ & $\tau D_{\mathrm{G}} / N, \times 10^{2}$ \\
\hline 8 & 6.00 & 14.6 & 5.1 & 4.87 & 13.7 & 4.4 & 3.63 & 12.9 & 3.5 \\
\hline 12 & 3.85 & 6.1 & 5.2 & 2.68 & 5.9 & 3.8 & 1.69 & 3.7 & 3.8 \\
\hline 16 & 3.13 & 3.8 & 5.1 & 1.83 & 2.7 & 4.3 & 1.10 & 2.0 & 3.4 \\
\hline 20 & 2.43 & 2.4 & 5.1 & 1.28 & 1.8 & 3.6 & 0.75 & 1.2 & 3.2 \\
\hline 24 & 2.04 & 1.8 & 4.8 & 1.08 & 0.94 & 4.8 & 0.57 & 0.57 & 4.2 \\
\hline 32 & - & - & - & 0.61 & 0.52 & 3.7 & 0.32 & 0.31 & 3.2 \\
\hline 40 & - & - & - & 0.45 & 0.32 & 3.5 & 0.21 & 0.15 & 3.5 \\
\hline
\end{tabular}
$c=0.0, N=32$ and $c=0.25, N=32$.

Table I. Values of $D_{\mathrm{G}}, \tau$, and $\tau D_{\mathrm{G}} / N$ 


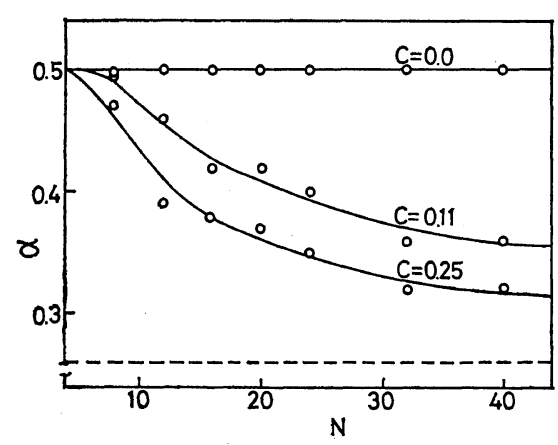

Figure 10. The quantity $\alpha$ is plotted against the molecular weight $N$.

to some definite value which depends on the concentration. Then we can hardly expect that, for sufficiently large molecular weight, $\alpha$ tends to 0.25 . We consider that this discrepancy is the consequence of the weakness of the reptation model. In the reptation model, the Brownian motion of each monomer is described rather crudely. For example, the motion perpendicular to the tube is completely neglected. Therefore the discrepancy is an approval one. This point will be discussed further in the following section.

\section{DISCUSSION}

In the present work the stochastic motion of a chain in fixed obstacles are investigated by changing the chain length and the concentration of the obstacles.

Let us first consider the region where the reptation model is supposed to be a good one. In our computer experiment, this corresponds to the region of $N \geqslant 20$ and $c=0.25$. It is found that the correlation functions $\left\langle\left(\boldsymbol{R}_{G}(t)-\boldsymbol{R}_{G}(0)\right)^{2}\right\rangle$ and $\langle\boldsymbol{P}(t) \cdot \boldsymbol{P}(0)\rangle$ agree well with those predicted by the de-Gennes' theory if some minor revisions are made. The agreement is fairly good not only for the molecular weight dependency but also for the absolute numerical values, which can be checked by eq 48 . Some important points observed by the experiments are as follows.

(1) The mean square displacement of the center of mass is not proportional to $t$ but is written as $A+4 D_{\mathrm{G}} t$, where the constant term $A$ does not vanish in general for $c \neq 0.0$.

(2) The fluctuation of the tube length cannot be neglected in calculating the correlation function of the end-to-end vector.

(3) The parameters $\bar{\rho}, b$ and $\Delta$ depend on the concentration of the obstacles, therefore the quantities $D_{\mathrm{G}} N^{2}$ and $\tau / N^{3}$ are the function of the concentration of the obstacles.

Although these points were not referred in de-Gennes' papers, they can be predicted based upon the reptation model as was discussed in the foregoing section.

However a serious discrepancy arises in the correlation function $\left.\left\langle\boldsymbol{R}_{\mathrm{c}}(t)-\boldsymbol{R}_{\mathrm{c}}(0)\right)^{2}\right\rangle$, especially in its short time behaviour. It is found that $\left\langle\left(\boldsymbol{R}_{\mathrm{c}}(t)-\boldsymbol{R}_{\mathrm{c}}(0)\right)^{2}\right\rangle$ is not proportional to $t^{1 / 4}$ but to $t^{\alpha}(1 / 4 \leqq \alpha \leqq 1 / 2)$. This discrepancy is, we suppose, a consequence of the weakness included in the reptation model. In the reptation model the chain is assumed to move only along the virtual tube. However such a description is good only for a long time scale, since only in such a time scale, can the deviation of the monomer from the virtual tube be neglected compared to the displacement along the tube. In the short time scale the deviation of the monomer from the tube cannot be neglected. The reptation model is too crude to describe such a motion, and another model is needed to discuss the motion of the monomer in the short time scale $\left(t \lesssim T_{\mathrm{d}}\right)$. We suppose that the monomer can move quite freely in such a time scale of observation.

In conclusion, for a sufficiently long chain or for sufficiently dense obstacles, the reptation model is fairly good for the "slow relaxation phenomena" but not so good for the "rapid relaxation phenomena." The slow relaxation phenomena are those whose characteristic time increases as the molecular weight increases. For such phenomena the global conformation of the chain is important and therefore the results are independent of the mechanism of the reptation. In fact it can be shown that the results of the de-Gennes' theory can be completely derived based upon the bead-spring model moving only along the virtual tube.

Next let us consider the case of intermediate concentration of the obstacles. The important question to be discussed is what is the condition that the reptation model can be applied. Our 


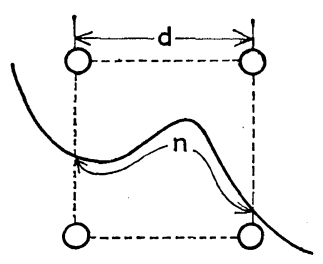

Figure 11. Local conformation of the chain. The mean number of the monomer contained in the "unit cell" of the piles is equal to $n \cong d^{2} / b^{2}$.

consideration for this question is as follows. Let $d$ be the mean interval between the piles. It is related to the density of the piles as $d=c^{-1 / 2}$. Consider a polymer chain penetrating the unit cell of the piles (see Figure 11). Let $n$ be the mean number of monomers contained in the unit cell. Then the condition that the chain is regarded as confined in the virtual tube can be written as

$$
N \gg n
$$

On the other hand $n$ can be related to $d$ by the following relation

$$
\begin{aligned}
d^{2} \cong & \text { (mean square distance of the subchain } \\
& \quad \text { consisting of } n \text { monomers }) \\
= & n a^{2}
\end{aligned}
$$

Then from eq 51 and 52, we obtain

$$
N a^{2} c \gg 1
$$

Let us now return to the experimental results. From Figures 5, 7 and 8 it is seen that the reptation model can be applied for $N \gtrsim 20$ in the case $c=0.25$. Therefore the above criterion can be written as

$$
N c \gtrsim 20 \times 0.25=5
$$

From this it is concluded that for case $c=0.11$, the molecular weight is not large enough for the reptation model to be applied even when $N=40$. This conclusion is qualitatively in consistence with the behaviour of the curve of $c=0.11$ in Figures 5, 7 and 8 . Firm experimental evidence of this criterion, however, is not obtained because of the reason that the simulation for the long polymer chain rapidly becomes difficult. This point will be investigated further in a future report.

Finally let us consider the motion of the chain for the case of intermediate concentrations. It seems at this stage that the theoretical investigation of the motion for this case is most difficult since we must take into account the topological restriction in a more appropriate way. However the results of such an investigation may not be very interesting, since they are between those two of the Rouse theory and de-Gennes'. The computer experiments shows that the transition occurs rather smoothly from the Rousetype motion to the de-Gennes-type motion. No abrupt change appears. Therefore we presume that, based upon the reptation model, we will not be able to explain the abrupt change of the molecular weight dependency of the viscosity observed in the real undiluted polymer solusions. The change will be the consequence of some collective motion of the polymer chains.

Acknowledgement. The author wishes to thank Dr. Akira Ito for his helpful advices on the computer programs. $\mathrm{He}$ also expresses to his thanks to Prof. Koji Okano and Prof. Yasaku Wada for their valuable suggestions, and to Prof. Shigeo Iwayanagi and Dr. Yasuaki Kawamura for kindly permitting the author to make use of the computer system of the Institute of Physical and Chemical Research.

\section{APPENDIX I}

In this section we will show that eq 34 can be derived from the reptation model. The calculation is a small extension of the de-Gennes' theory and similar notations are used. Consider a weak uniform time-dependent force $\boldsymbol{F}(t)$ applied to the polymer chain in the networks. The velocity of the center of mass is given by

$$
\begin{aligned}
\dot{\boldsymbol{R}}_{\mathrm{G}}(t) & =\frac{1}{N} \sum_{n=1}^{N} \dot{\boldsymbol{R}}_{n}(t), \\
& =\frac{1}{N} \sum_{n=1}^{N} \boldsymbol{b}\left(s_{n}(t)\right) \cdot \dot{s}_{n}(t)
\end{aligned}
$$

where $\boldsymbol{b}(s)$ is a vector of magnitude $b$, tangential to the virtual tube and $s_{n}$ is the curvelinear length along the tube between the first and the $n$-th monomer. As is shown in de-Gennes' paper $\dot{s}_{n}(t)$ is given by

$$
s_{n}(t)=\sum_{m=1}^{N} \int_{-\infty}^{t} \mathrm{~d} t^{\prime} \beta_{n m}\left(t-t^{\prime}\right) \boldsymbol{F}\left(t^{\prime}\right) \cdot \boldsymbol{b}\left(s_{m}\left(t^{\prime}\right)\right) / N
$$


with

$$
\begin{aligned}
& \beta_{n m}= \frac{\bar{\rho} b^{2} \Delta}{a k_{\mathrm{B}} T}\left[\frac{\delta_{+}(t)}{N}+\frac{2}{N} \sum_{p=1}^{N} \cos \frac{p \pi n}{N} \cos \frac{p \pi m}{N}\right. \\
&\left.\times\left\{\delta_{+}(t)-\frac{1}{\tau_{p}} \mathrm{e}^{-t / \tau_{p}}\right\}\right] \\
& \tau_{p}=\frac{a^{2}}{\pi^{2} \Delta} \frac{N^{2}}{p^{2}}
\end{aligned}
$$

The above calculation is concerned with a polymer chain reptating in a given fixed tube: To obtain the mean velocity, we must take the average with respect to the conformation of the tube, that is, with respect to $\boldsymbol{b}(s)$. Substituting eq 56 into eq 55 , we must calculate the average

$$
\left\langle\boldsymbol{b}\left(s_{n}(t)\right) \boldsymbol{b}\left(s_{m}\left(t^{\prime}\right)\right)\right\rangle
$$

Here we adopt the following approximation, which is verified from the result.

$$
\begin{aligned}
\left\langle\boldsymbol{b}\left(s_{n}(t)\right) \boldsymbol{b}\left(s_{m}\left(t^{\prime}\right)\right)\right\rangle & \cong\left\langle\boldsymbol{b}\left(s_{n}\left(t^{\prime}\right)\right) \boldsymbol{b}\left(s_{m}\left(t^{\prime}\right)\right)\right\rangle \\
& =\frac{b^{2}}{3} \mathbf{I} \delta_{n m}
\end{aligned}
$$

where $\mathbf{I}$ is the unit tensor. Substituting eq 56 and 57 into eq 55 and using eq 59, we obtain

$$
\begin{aligned}
\dot{\boldsymbol{R}}_{\mathrm{G}}(t) & =\frac{1}{3 N^{2}} \sum_{m=1}^{N} \int_{-\infty}^{t} \mathrm{~d} t^{\prime} \beta_{n m}\left(t-t^{\prime}\right) \boldsymbol{F}\left(t^{\prime}\right) \\
& \equiv \int \mathrm{d} t^{\prime} B\left(t-t^{\prime}\right) \boldsymbol{F}\left(t^{\prime}\right)
\end{aligned}
$$

According to the Kubo formula

$$
\left\langle\dot{\boldsymbol{R}}_{\mathrm{G}}(t) \cdot \dot{\boldsymbol{R}}_{\mathrm{G}}(0)\right\rangle=3 k_{\mathrm{B}} T B(t)
$$

Therefore

$$
\begin{aligned}
\left\langle\left(\boldsymbol{R}_{\mathrm{G}}(t)\right.\right. & \left.\left.-\boldsymbol{R}_{\mathrm{G}}(0)\right)^{2}\right\rangle \\
= & 2 \int_{0}^{t} \mathrm{~d} t_{1} \int_{0}^{t_{1}} \mathrm{~d} t_{2}\left\langle\dot{\boldsymbol{R}}_{\mathrm{G}}(t) \cdot \dot{\boldsymbol{R}}_{\mathrm{G}}(0)\right\rangle \\
= & \frac{2}{N^{2}} \int_{0}^{t} \mathrm{~d} t_{1} \int_{0}^{t_{1}} \mathrm{~d} t_{2} \sum_{n, m=1}^{N} \beta_{n m}\left(t_{2}\right)
\end{aligned}
$$

Substituting eq 57 into eq 62 we obtain after some calculation

$\left.\left\langle\boldsymbol{R}_{G}(t)-\boldsymbol{R}_{G}(0)\right)^{2}\right\rangle=\frac{2 \bar{\rho} b^{2} \Delta}{N^{2} a}\left[t+\sum_{p} \tau_{p}\left(1-\mathrm{e}^{-t / \tau} p\right)\right]$
For $t \geqq \tau_{1}$ eq 63 is approximated as

$$
\begin{gathered}
\left\langle\left(\boldsymbol{R}_{G}(t)-\boldsymbol{R}_{\mathrm{G}}(0)\right)^{2}\right\rangle=A+6 D_{\mathrm{G}} t \\
A=2 \frac{\bar{\rho} b^{2} \Delta}{N^{2} a} \sum_{p} \tau_{p}=\frac{1}{3} \bar{\rho} b^{2} a \\
D_{G}=\frac{\bar{\rho} b^{2} \Delta}{3 N^{2} a}
\end{gathered}
$$

The above calculation is for the three-dimensional stochastic motion. For the case of the two-dimensional motion, the result is

$$
\begin{gathered}
\left\langle\left(\boldsymbol{R}_{G}(t)-\boldsymbol{R}_{G}(0)\right)^{2}\right\rangle=A+4 D_{\mathrm{G}} t \\
A=\frac{1}{3} \bar{\rho} b^{2} a \\
D_{G}=\frac{\bar{\rho} b^{2} \Delta}{2 N^{2} a}
\end{gathered}
$$

Note that $A$ is independent of $N$, this fact is observed in our computer experiments.

\section{REFERENCES}

1. J. D. Ferry, "Viscoelastic Properties of Polymers," Wiley, New York, N.Y., 1970.

2. F. Bueche, J. Chem. Phys., 20, 1959 (1952), 25 599 (1956).

3. W. W. Grassley, ibid., 43, 2669 (1965); 47, 1942 (1967).

4. Y. Chikahisa, J. Phys. Soc. Jap., 19, 92 (1964).

5. A. J. Chompff and J. A. Duiser, J. Chem. Phys., 45, 1505 (1966).

6. A. J. Chompff and W. Prins, ibid., 48, 235 (1968).

7. S. Hayashi, J. Phys. Soc. Jap., 27, 1583 (1969).

8. M. Yamamoto, R.P.P.J., XIV (1973) to be published.

9. M. Yamamoto, J. Phys. Soc. Jap., 11, 413 (1956).

10. S. F. Edwards, Proc. Phys. Soc., 91, 513 (1967); J. Phys. A, 1, 15 (1968).

11. P. G. de-Gennes, J. Chem. Phys., 55, 572 (1971).

12. P. G. de-Gennes, Physics, 3, 37 (1967).

13. P. H. Verdier and W. H. Stockmayer, J. Chem. Phys., 36, 227 (1962).

14. P. H. Verdier, ibid., 52, 5512 (1970). 